\title{
Neuronal Modifications During Visuomotor Association Learning Assessed by Electric Brain Tomography
}

\author{
Elke Praeg, ${ }^{* \dagger} \dagger$ Michaela Esslen, ${ }^{*}$ Kai Lutz, ${ }^{*}$ and Lutz Jancke*
}

\begin{abstract}
Summary: In everyday life specific situations need specific reactions. Through repetitive practice, such stimulus-response associations can be learned and performed automatically. The aim of the present EEG study was the illustration of learning dependent modifications in neuronal pathways during short-term practice of visuomotor associations. Participants performed a visuomotor association task including four visual stimuli, which should be associated with four keys, learned by trial and error. We assumed that distinct cognitive processes might be dominant during early learning e.g., visual perception and decision making. Advanced learning, however, might be indicated by increased neuronal activation in integration- and memory-related regions. For assessment of learning progress, visual- and movement-related brain potentials were measured and compared between three learning stages (early, intermediate, and late). The results have revealed significant differences between the learning stages during distinct time intervals. Related to visual stimulus presentation, Low Resolution Electromagnetic Brain Tomography (LORETA) revealed strong neuronal activation in a parieto-prefrontal network in time intervals between $100-400 \mathrm{~ms}$ post event and during early learning. In relation to the motor response neuronal activation was significantly increased during intermediate compared to early learning. Prior to the motor response (120-360 ms pre event), neuronal activation was detected in the cingulate motor area and the right dorsal premotor cortex. Subsequent to the motor response (68$430 \mathrm{~ms}$ post event) there was an increase in neuronal activation in visuomotor- and memory-related areas including parietal cortex, SMA, premotor, dorsolateral prefrontal, and parahippocampal cortex. The present study has shown specific time elements of a visuomotor-memory-related network, which might support learning progress during visuomotor association learning.
\end{abstract}

Key words: Association learning; Integration; Neuronal modifications; EEG; LORETA.

\section{Introduction}

A specific feature of the human brain is the capacity to modify neuronal pathways even during short-term learning. In order to react to sensory information with fast and adequate behavior, situation-dependent associations between sensory stimuli and motor responses are learned on the basis of repetitive practice and adequate feedback. One example is acquiring an association between a visual cue indicating a musical sound (e.g., a grapheme indicating the tone /a/) and a particular finger movement (e.g., pressing a specific key on a piano keyboard). Consolidation of the acquired association requires repeated activation of the appropriate motor response in combination with inhibition of inap-

*Department of Neuropsychology, Institute of Psychology, University of Zurich, Treichlerstrasse 10, CH-8032 Zurich, Switzerland.

Accepted for publication: September 11, 2006; Published Online: November 302006.

${ }^{\dagger}$ Correspondence and reprint requests should be addressed to Elke Praeg (M.S.), Center for Noninvasive Brain Stimulation, Beth Israel Deaconess Medical Center, Harvard Medical School, 330 Brookline Avenue, Boston, MA 02215, U.S.A. E-mail: epraeg@ bidmc.harvard.edu, l.jaencke@psychologie.unizh.ch

Copyright (c) 2006 Springer Science + Business Media, Inc. propriate motor responses. This is particularly important when different responses are to be associated with different sensory stimuli that have distinctive features in common. The contextually correct stimulus-response association should be activated whereas other responses based on similar visuomotor associations should be actively inhibited (Karni et al. 1998; Garavan et al. 1999; Jackson et al. 1999). Thus, selective associations of sensory stimuli with adequate motor responses are inseparably combined with activation and inhibition of specific vision- and movement-related brain regions.

Brain imaging studies, human lesion studies and animal single cell recordings provided evidence that a neuronal network of prefrontal, premotor, parietal and striatal areas is involved in visuomotor association learning (Johnson et al. 1996; Wise et al. 1997; Matelli et al. 2000; Toni et al. 2001; Toni et al. 2002b; Matsumoto et al. 2003; Brasted et al. 2004). Although the brain areas, which might be involved in controlling and processing visuomotor associations are often investigated, the temporal dynamics of the neuronal modifications during visuomotor association learning are less clearly understood. A few fMRI-studies have concentrated on this issue in human beings (Toni et al. 2001; Thoenissen et al. 2002). These studies revealed a learning-related modification in 
hemodynamic response during association learning in a temporo-prefrontal circuit that included medial temporal cortex, parahippocampal gyrus, hippocampus and caudate nucleus. In a follow-up study (Toni et al. 2002a), learning-dependent amplification of effective connectivity was demonstrated in temporo-striatal and frontostriatal circuits but no alterations in temporo-frontal and parieto-frontal couplings. Thus, a shift in the recruitment of occipito-parietal areas to frontal areas seems to be evident with increasing practice.

In order to overcome the poor time resolution of present brain imaging studies, EEG studies are used for better time resolution. However, only a few EEG studies have examined changes in cortical activation associated with visuomotor learning. Staines and collegues (Staines et al. 2002) calculated ERPs during learning of novel movements in a visually cued motor task. At presentation of a visual cue, a fronto-central network was active during the initial learning phase but this activation diminished with ongoing practice. During late compared to early learning, there was an increase in several ERP amplitudes following movement onset. In addition, the latencies of these amplitudes were shorter over centroparietal sites, especially during late learning. It was hypothesized that a fronto-parietal network becomes increasingly more involved during later learning stages, thus indicating most efficient sensorimotor integration.

The present study was designed to investigate visuomotor association learning in order to delineate the time dependent modification of an integration-related network. We conducted the ERP study to achieve significant differences between topographical differences of ERP activity of three learning stages. Based on the topographical distribution of the ERPs, intracerebral sources of electrical activity was estimated by using LORETA (Low Resolution Electric Brain Tomography, Pascual-Marqui et al. 2002). The aim of the present study was twofold: (1) to elucidate the temporal dynamics of learning-dependent changes in cortical activation by delineating significant time epochs of current density changes and (2) to calculate the intracerebral sources of neuronal activation changes.

Based on previous studies of visuomotor association learning, we hypothesize that early visuomotor learning is controlled by distinct processes including visual perception, decision making, working memory and motor execution. In contrast, during advanced learning stages neuronal modifications might strengthen integrationand memory-related regions. The present study utilizes the fact that the EEG method permits separate examination of brain responses evoked by visual cues (visual evoked potentials related to visual cues) and those connected with motor responses (motor related potentials related to motor responses). By using LORETA it is possible to illustrate neuronal modifications that are based on the data of all electrodes instead of analysis of single electrodes. Additionally, we are not restricted to time intervals of previously described prominent peaks but specifically describe learning-related activation differences also in time epochs between those peaks.

\section{Methods}

\section{Participants}

Twenty-one healthy volunteers participated in the study. Formal hand preference testing with the AnnettHandedness-Questionnaire (Annett, 1992) confirmed that all subjects were consistently right-handed. They were informed about the study and gave written informed consent for participation. Four subjects were excluded from the final analysis because of muscle artefacts contaminating the EEG data. A further subject was excluded because of self-reported dyslexic problems. Thus, data of sixteen subjects ( 8 women, 8 men; $20-37$ years of age, mean age 24.6-4.3 SD) were entered for final analysis. The Ethics Committee of the University of Zurich gave approval for the study.

\section{Experimental Task}

Subjects performed a visuomotor association task that changed to a choice reaction task once subjects had learned the correct associations. During each experimental block they learned to associate four fingers (and corresponding buttons) with four different visual stimuli (following the "imperative stimuli") of the same category. Button presses were conducted with the dominant right hand using one of four fingers (index, middle, ring, and little). All four fingers were placed over the "J", "K", "L", "Ö" buttons on a German keyboard (corresponding to "J", "K", "L", "; " on a standard QWERTY computer keyboard). Three categories of well-known visual stimuli were presented. These were either the letters " $\mathrm{A}$ ", " $\mathrm{B}$ ", " $\mathrm{C}$ " and " $\mathrm{D}$ ", arrows pointing in four different directions, that is, "up", "down", "left" and "right", and the digits " 2 ", " 3 ", " 4 " and " 5 ". We chose these stimuli because well-educated subjects should be familiar with these, thereby reducing object recognition and visual processing times as much as possible. As our area of special interest, one aim was to minimize the visual processing steps in order to isolate the different learning-dependant cortical activations associated with visuomotor association learning. Letters and digits were presented in "Times New Roman" using font size 70 (size [height $\mathrm{x}$ width] letters: $2.2 \times 2 \mathrm{~cm}$, digits: $2.7 \times$ $1.4 \mathrm{~cm}$ ). The size of the upright arrows were: $1.7 \times 0.7 \mathrm{~cm}$, and vice versa for left/right. The size of the feedback signals were tick: $1.3 \times 1.8 \mathrm{~cm}$ and cross: $1.3 \times 1.3 \mathrm{~cm}$. The background was kept in grey (RGB: 192, 192, 192) 
to minimize background radiation in the dimmed room. Fixation cross, imperative stimuli and visual feedback were presented in black (RGB: 0, 0,0) in the centre of a computer screen.

During execution of an experimental block each imperative stimulus was associated with a specific finger of the right hand (e.g., in the first block of the category "letters", " $\mathrm{B}$ " was associated with index, " $\mathrm{D}$ " with middle, " $A$ " with ring, and " $C$ " with little finger). Direct linearly ordered associations like "index to $\mathrm{A}^{\prime}$, "middle to $\mathrm{B}^{\text {", }}$ "ring to $C$ " and "little to $D$ " were avoided to ensure that no pre-learned orders could be used. Every category of imperative stimuli was used twice, resulting in six blocks. The associations of the imperative stimuli to the corresponding buttons changed after every block independently of the category. The sequence of the blocks was randomized. Each block of the experiment included 160 pseudo-randomized trials and lasted approximately 8 minutes. Every subject performed all six blocks, including three types of stimuli, each with two different stimulus-response combinations.

Each trial started with presentation of the fixation cross for $1000 \mathrm{~ms}$ followed by one of four imperative stimuli. Subjects were instructed to respond with one button press as fast as possible after appearance of the imperative stimulus. They learned the correct association of the imperative stimuli to the buttons by trial and error. Immediately after motor response, the imperative stimulus disappeared and was followed by a blank screen for $500 \mathrm{~ms}$. Visual feedback was then presented for $500 \mathrm{~ms}$ to inform whether the chosen finger was used correctly or incorrectly. In case of correct responses, the "correct" sign (" $\sqrt{ }$ " or "tick") appeared in the centre of the screen, otherwise an "incorrect" sign (' $x$ ' or "cross") followed. Immediately after visual feedback presentation a new trial started with presentation of the fixation cross. If there was no response, the imperative stimulus disappeared after $3000 \mathrm{~ms}$ and a new trial started (Fig. 1). After finishing each block, a short break was introduced allowing the subjects to relax. Dependent on reaction times and individual breaks subjects needed approximately $60 \mathrm{~min}$ for execution of the experiment.

Subjects were comfortably seated in a chair situated in a dimmed room, in front of a desk, with the chin placed on a chin rest for the entire duration of the experiment. The distance between screen and eyes was kept constant at $90 \mathrm{~cm}$. To avoid auditory disturbances subjects used earplugs. Stimulus delivery and registration of subjects' responses were controlled by "Presentation" software (Neurobehavioral Systems, Albany, CA, USA).

For the purpose of investigating functional changes in brain activation patterns over time, three learning stages were defined. We assumed that improvements in performance would be reflected in corresponding improvements in reactions times and error rates. Learning stages were defined according to the empirically measured error rates only. For this, a criterion of $90 \%$ correct responses $(10 \%$ error rate) was used to indicate welllearned visuomotor association and the end of initial "early" learning. "Intermediate" learning was defined as the stage following "early" learning, and "late" learning as the following and final stage of each block. Each learning stage comprised the same number of trails.

\section{EEG Recording}

EEG was recorded continuously using the international 10-10 system with 30 electrodes $[\mathrm{Fp} 1 / 2, \mathrm{~F} 3 / 4$, $\mathrm{F} 7 / 8, \mathrm{Fz}, \mathrm{FT} 7 / 8, \mathrm{FC} 3 / 4, \mathrm{~T} 7 / 8, \mathrm{C} 3 / 4, \mathrm{Cz}, \mathrm{TP} 7 / 8, \mathrm{CP} 3 / 4$, $\mathrm{CPz}, \mathrm{P} 7 / 8, \mathrm{P} 3 / 4, \mathrm{Pz}, \mathrm{O} 1 / 2, \mathrm{Oz}$, referred to $\mathrm{FCz}$ (reference)] and two Electrooculogram (EOC) electrodes below the outer canthus of each eye. We used a standard EEG cap ("Easy Cap", FMS Falk Minow Services, Herrsching-Breitbrunn, Germany) and sintered $\mathrm{Ag} / \mathrm{AgCl}-$ electrodes. Electrode impedances were kept below $5 \mathrm{k} \Omega$. EEG data was recorded with a sampling rate of $500 \mathrm{~Hz}$ and bandpass filter $(0.5-70 \mathrm{~Hz})$ using BrainProducts amplifiers (BrainProducts, Munich, Germany). A written introduction was presented on the computer screen, describing the forthcoming experimental task.
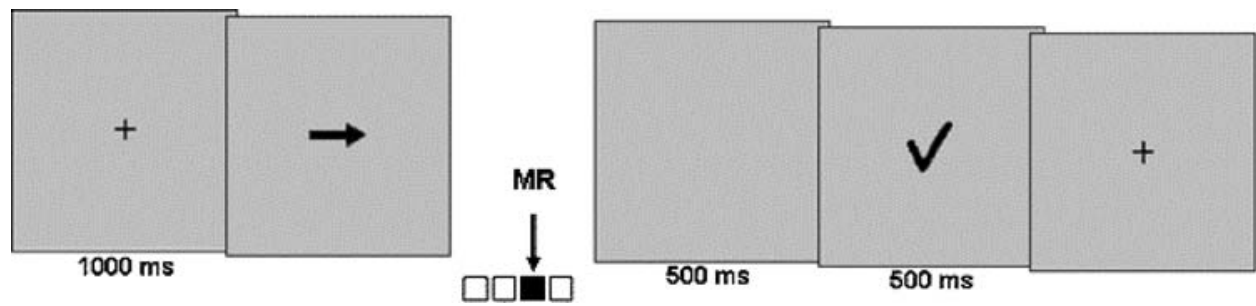

Figure 1. Paradigm of the visuomotor association task. A fixation cross was presented for $1000 \mathrm{~ms}$ at the beginning of each trial to avoid eye-movements. Subjects had to press one of four buttons as fast as possible (motor response) after appearance of one of four imperative stimuli (here: arrow, direction: right). By onset of motor response the visual imperative stimulus disappeared and a blank screen $(500 \mathrm{~ms})$ was presented. Following the motor response a visual feedback was presented (here: correct) for $500 \mathrm{~ms}$. A new trial started with the presentation of the fixation cross. 


\section{EEG Data Pre-Processing}

Using Brain Vision Analyzer Software (Brain Products, Munich, Germany), the EEG recordings were digitally band pass filtered to $0.5-30 \mathrm{~Hz}$. For automatic artefact rejection an amplitude criterion of $\pm 100 \mu \mathrm{V}$ was used for all channels at any time point. All sweeps with eye blinks or eye movements were excluded manually on visual inspection. The remaining sweeps were transformed to average reference. All blocks of the experiment were segmented into three learning stages according to the accuracy of correct responses. Event-related potentials (ERP) were calculated time-locked (a) to the imperative stimulus (visual-evoked potential, VEP) and (b) to correct motor response (movement-related potential, MRP), for each individual subject and for each learning stage. Time epochs (a) from onset of the imperative stimulus to $500 \mathrm{~ms}$ after the imperative stimulus onset and (b) from $400 \mathrm{~ms}$ before motor response to $500 \mathrm{~ms}$ after execution were included in further analysis.

\section{ERP Statistics}

Grand averages of VEPs and MRPs were computed to visualize alterations between learning stages and to identify prominent amplitudes. It is generally well established that peak amplitudes indicate time elements of prominent activation or illustrate significant changes interpreted as changes in different patterns of neuronal processing. It is obvious, however, that brain activation is not restricted in processing these few events and that neuronal activation does not diminish between the peaks. We expected functional changes in visuomotor association processing to take place during the course of the whole time epochs of visuomotor processing. Based on this assumption and contrary to earlier EEG studies, we did not exclusively analyse ERPs taken from specific electrodes nor did we statistically analyse prominent ERP components. However, for the benefit of comparison with previous EEG studies, in the results section we describe prominent peaks of the central line, while we focus in the discussion on differences in the scalp topography between different learning stages at particular time points.

For illustration of neuronal modifications related to VEPs and MRPs, we evaluated activation differences between the learning stages. Each interval was associated with a specific ERP scalp map and an associated estimation of current density distribution. We compared differences between the ERP scalp maps for the different learning stages as a function of time (Esslen et al. 2004; Lehmann et al. 1987). Statistical significance for each pair of maps was assessed nonparametrically using a randomization test (Manly 1997). This procedure, hereafter called "topographic analysis of variance" (TANOVA), computes the overall dissimilarity between ERP scalp topographies (Strik et al. 1998). For this, the vectors defined by $n$ scalp electrodes $(n=32)$ were conceptualized. TANOVA and dependent samples $t$-tests were used to compute the dissimilarity at each of the timepoints of the ERPs obtained for the different learning stages while performing random permutations (1000) to correct for false positives (Nichols et al. 2002). Prior to submission to TANOVA, the average ERP segments of all participants were average-referenced and transformed to a global field power of 1 . This procedure ensures that the dissimilarity is not influenced by higher activity across the scalp in one of the conditions. Based on these TANOVAs time segments of significantly different topographic ERP maps between the learning stages were revealed; these are referred to as "time elements." The statistical criterion for identifying a significant map difference was set at $p<0.05$ (corrected for multiple comparisons). Dependent on these significantly different time elements, the time epochs of the VEP $(500 \mathrm{~ms})$ and MRP $(900 \mathrm{~ms})$ were segmented in corresponding time elements individually for each subject and learning stage. In order to visualize the significantly different ERP maps these differences were presented as $t$-maps in Figs. 4 and 5.

\section{Source Modelling}

We used LORETA to localize neuronal activation changes between learning stages. This method has been extensively validated in a number of studies (Vitacco et al. 2002; Sinai et al. 2003; Esslen et al. 2004; Mulert et al. 2004b). In short, LORETA assumes that the smoothest of all current density distributions is most plausible and on this basis identifies a particular current density distribution (Pascual-Marqui et al. 2002). This "smoothness assumption" of LORETA relies on the coherent firing of neighbouring cortical neurons during stimulus processing (Gray et al. 1989; Silva et al. 1991) and can therefore be seen as a physiologically based constraint. The characteristic feature of the resulting LORETA solution is its relatively low spatial resolution, which is a direct consequence of the smoothness constraint. The solution space is restricted to cortical grey matter and hippocampi.

Based on the resulting time elements of the TANOVA analysis, LORETA images were calculated as the average of current density magnitude over all instantaneous LORETA images within the interval separately for each voxel. Localization inference was based on voxel-by-voxel $t$-tests of LORETA images among any of the three learning stages ("early" vs. "intermediate," and "intermediate" vs. "late" learning stages). Statistical significance was assessed nonparametrically with a randomization test (Nichols et al. 2002). Only those differences associated with a $p<0.05$ (corrected for multiple comparisons) will be described and interpreted. Given 
the limited spatial resolution provided by LORETA, the anatomical locations associated with significantly different neuronal activations during the different learning stages are illustrated in terms of the anatomical location estimated from the standard MNI brain provided by the Montreal Neurological Institute. The obtained LORETA images were compared on a voxel-wise basis for differences between the learning stages. The statistical comparisons were conducted using the randomization tests implemented in LORETA, resulting in conservative pairwise tests corrected for multiple comparisons.

\section{Analysis of Behavioural Data}

Behavioural data were examined with the measures reaction times (RT) and accuracy of correct responses. All data were checked for normal distribution and fulfilled the requirements for processing with parametrical tests. In addition, we also calculated $\eta^{2}$ because of the importance of reporting effect sizes unrelated to sample size (Cohen 1977). Because we calculated two repeated measurements ANOVAs (with three levels) a corrected $p$-value of $p=0.025(p=0.05 / 2)$ was used as statistical threshold. In case of a significant main effect subsequent Bonferroni-corrected post-hoc $t$-tests were calculated. For Bonferroni-correction the Holm procedure was used (Holm 1979). Before calculation of each repeated measurements ANOVA the variances were evaluated for deviations from homoscedasticiy. None was detected.

\section{Results}

Accuracy Scores, Definition of Learning Stages and Reaction Times

Analysis of the accuracy scores revealed that all subjects reached, on average, $90 \%$ correct responses in all conditions after 25 trials. Hence, we defined the first 25 trials as "early" leaning stage. After the first 25 trails no further increase in accuracy was evident. We assumed ongoing changes in cortical activation in advanced learning stages and therefore retained the predefined stages and used the middle 25 trials (trials 68 to 93) and the last 25 trials (trials 135 to 160) as "intermediate" and "late" learning stages, respectively. A one-way repeated measurements ANOVA revealed a highly significant result $\left[F(2,30)=105.96, p<0.001, \eta^{2}=0.88\right]$ and post-hoc tests shown a significant increase in accuracy score from early to intermediate learning but no further increase from intermediate to late learning (early vs. intermediate: $t=10.96, p<0.001$, intermediate vs. late: $t=-0.67, p=0.512$ n.s.; mean accuracy of correct responses $(\%) \pm \mathrm{SE}$; early: $76.42 \pm 1.83$; intermediate: $97 \pm 0.48$; late: $96.58 \pm 0.64$ ).

Data analysis of mean reaction times for each learning stage as one-way repeated measurements ANOVA (three levels: "early", "intermediate", and "late") revealed a highly significant learning effect $[F(2,30)=$ 23.08, $\left.p<0.001, \eta^{2}=0.61\right)$ ]. Subsequently performed post-hoc t-tests showed a significant decrease in reaction times from early to intermediate learning stage $(t=-5.7, p<0.001)$, but no significant change of reaction times from intermediate to late learning ( $t=$ $-1.8, p=0.092$, n.s.; mean reaction times [ms] \pm SE: "early" 881.4 \pm 44.3 ; "intermediate" $778.4 \pm 37.8$; "late" $759.1 \pm 35.3)$.

\section{Event-Related Potentials}

Grand averages of all visually evoked and movement-related potentials of correct responses for all subjects are shown for the central electrodes (Figs. 2 and 3). We obtained a typical scalp distribution of the VEP, with a prominent P100 at occipital electrodes and N100 peak at frontocentral electrodes (Fig. 2). Approximately 140-175 ms after stimulus onset there was a second positive peak (P2) at frontocentral (Fz, FCz, Cz) and centroparietal electrodes $(\mathrm{CPz}, \mathrm{Pz})$, and at approximately $200 \mathrm{~ms}$ an occipital negativity. A further peak appeared approximately $300 \mathrm{~ms}$ following visual onset (P300) at parietal electrodes $(\mathrm{CPz}, \mathrm{Pz})$ and a simultaneous N300 at frontal electrodes (Fz, FCz).

Grand average of MRP for the epoch, beginning $400 \mathrm{~ms}$ before to $500 \mathrm{~ms}$ after correct motor response, is presented in Fig. 3. During early learning a slow negative potential shift evolves at parietal and occipital regions ( $\mathrm{Pz}$ and $\mathrm{Oz}$ ) $400 \mathrm{~ms}$ before motor response and resulted in a weak positive peak $50 \mathrm{~ms}$ before motor response at $\mathrm{CPz}$ and Pz. A weak positive peak was detected at $\mathrm{Fz}$ and $\mathrm{FCz}$ approximately $200 \mathrm{~ms}$ before motor response. Negative peaks were presented precisely synchronous to correct motor response over Fz and FCz. $100 \mathrm{~ms}$ following motor response, positive peaks appeared over frontal electrodes (Fz, FCz and Cz). During advanced (intermediate and late) learning, increased positive deflections were detected $100 \mathrm{~ms}$ before the motor response over $\mathrm{FCz}, \mathrm{Cz}$ and $\mathrm{CPz}$. Following motor response, strong negative deflections were shown over parietal and occipital electrodes $(\mathrm{CPz}, \mathrm{Pz}$, and $\mathrm{Oz})$.

\section{Topographic Analysis of Variance (TANOVA)}

The TANOVA analysis of scalp current density distributions in relation to VEPs (VEP maps) revealed four time elements during which the current density distributions differ significantly between the early vs. the intermediate learning stage (112-154 ms, 206-286 ms, 


\section{Grand Average ( $n=16)$, visual-evoked potentials}
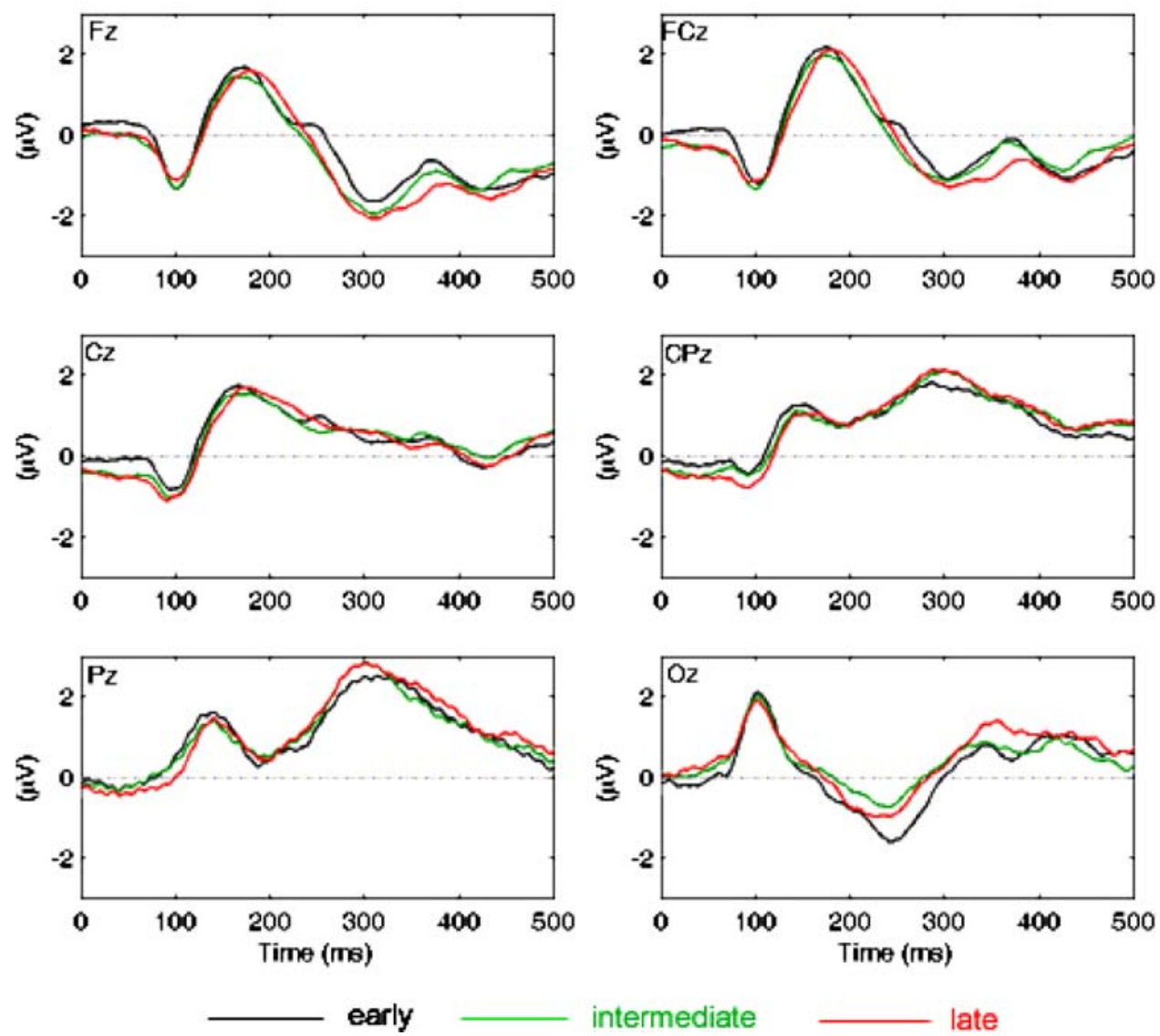

Figure 2. Grand average $(n=16)$ of visual-evoked potentials (VEPs). VEPs during three learning stages ("early" - black line, "intermediate" - green line, and "late" - red line) are shown time-locked to the onset of the imperative stimuli (time epoch: 0-500 ms following IS). VEPs are illustrated at central electrodes ( $F z, F C z, C z, C P z, P z$, and Oz). Positive values are up.

396-408 ms, 418-430 ms). Comparison of the VEP maps between the intermediate and late learning stage revealed one time element with a significant different current density distribution $(436-460 \mathrm{~ms})$. The TANOVA analysis of the scalp current density distributions in relation to the MRPs (MRP maps) revealed significant differences between early compared to intermediate learning stage at nine time elements (prior to motor response: $360-348 \mathrm{~ms}, 158-120 \mathrm{~ms}$, post motor response: $68-80 \mathrm{~ms}$, $168-206 \mathrm{~ms}, 220-232 \mathrm{~ms}, 252-298 \mathrm{~ms}, 304-320 \mathrm{~ms}, 370$ $380 \mathrm{~ms}, 410-430 \mathrm{~ms})$. There are no significant differences of MRP maps between intermediate and late learning stages.

\section{LORETA Analysis of the VEPs}

The TANOVA of the VEP maps revealed the aforementioned significant increase in activation during early compared to intermediate learning for four time elements after presentation of the visual imperative stimu- lus. LORETA (voxel-by-voxel $t$-test) localised the activation during these time elements as follows: superior part of the inferior parietal lobule (IPL) at $112-154 \mathrm{~ms}$; lateral orbitofrontal cortex (OFC) slightly extending into the lateral inferior frontal gyrus (IFG) at 206-286 ms; and, postcentral gyrus within secondary somatosensory cortex (SII) at 396-408 ms. During intermediate learning compared to early learning, activation increased significantly at the time element $418-430 \mathrm{~ms}$ and LORETA localised this to the left OFC comprising the gyrus rectus and right middle frontal gyrus. For a relatively late time element (436-460 ms) following the imperative stimulus we obtained increased neuronal activation for late vs. intermediate learning within the right superior frontal gyrus. Table I listed the time elements of local maxima with associated localised neuronal activation in Talairach and Tournoux coordinates. Corresponding Fig. 4 illustrated LORETA images at the calculated time elements as well as the scalp topography differences (as $t$-maps) for these time elements. 


\section{Grand Average ( $n=16)$, motor-related potentials}
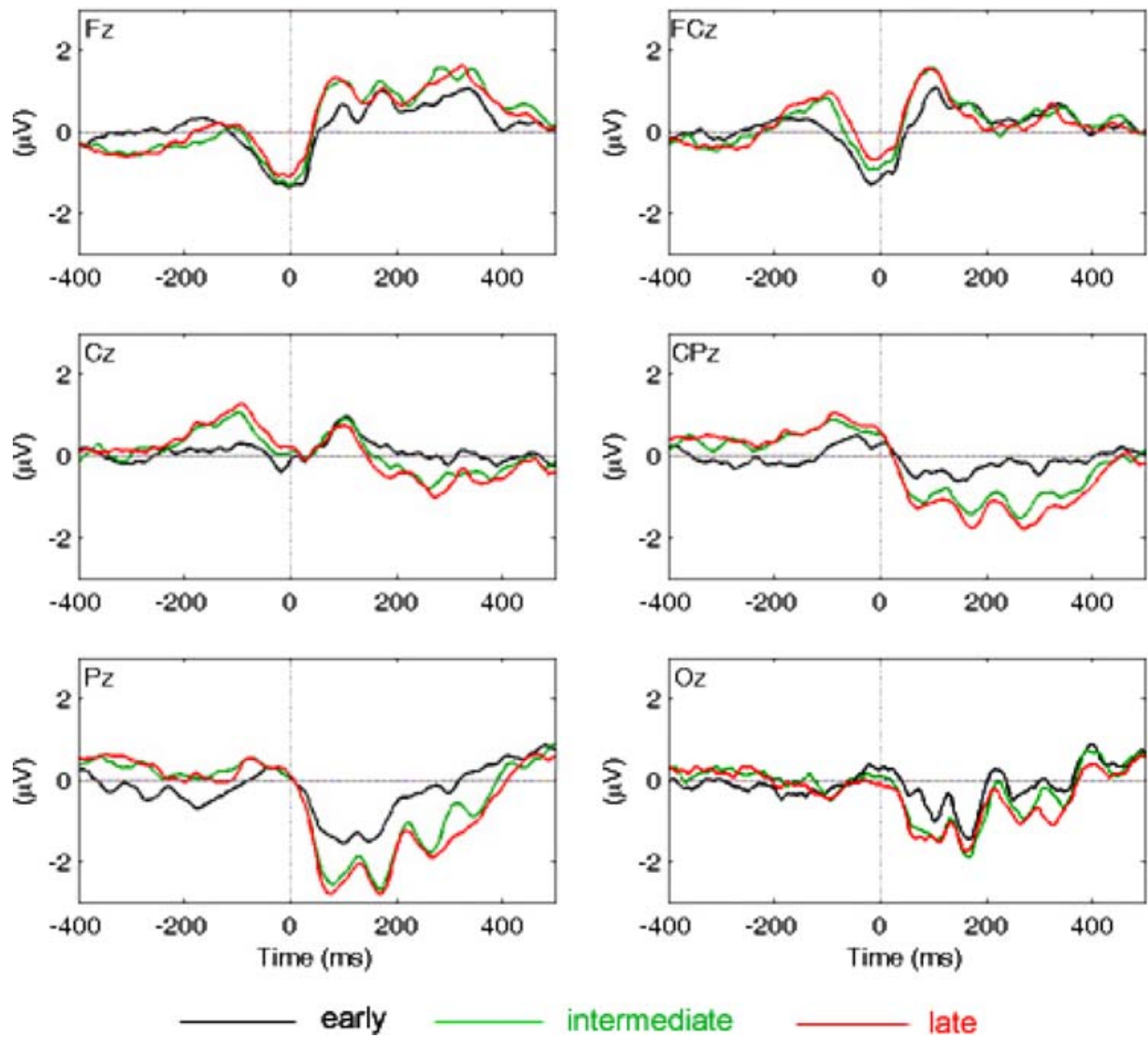

Figure 3. Grand averages $(n=16$ ) of the movement-related potentials (MRPs). MRPs during three learning stages ("early" - black line, "intermediate" - green line, and "late" - red line) are shown time-locked to correct motor response (time epoch: 400 before to $500 \mathrm{~ms}$ following motor response). Vertical dotted line indicates the time point of motor response. MRPs are illustrated at central electrodes ( $F z, F C z, C z, C P z, P z$, and $\mathrm{Oz}$ ). Positive values are up.

Table I. LORETA analysis of the VEPs: Significant differences in brain areas between learning stages ("early" vs. "intermediate" and "intermediate" vs. "late" learning)

\begin{tabular}{|c|c|c|c|c|c|c|}
\hline \multirow[b]{2}{*}{ Time element (in ms) } & \multirow[b]{2}{*}{ Functional anatomic area } & \multirow[b]{2}{*}{ BA } & \multirow[b]{2}{*}{ Contrast } & \multicolumn{3}{|c|}{ Tal coordinates } \\
\hline & & & & $x$ & $y$ & $z$ \\
\hline (A) 112-154 & (L) Inferior parietal lobule & 40 & $\mathrm{E}>\mathrm{I}$ & -52 & -53 & 50 \\
\hline \multirow[t]{3}{*}{ (B) 206-286 } & (L) Inferior frontal gyrus & 11 & $\mathrm{E}>\mathrm{I}$ & -24 & 31 & -20 \\
\hline & Medial frontal gyrus & 10 & & -10 & 45 & 8 \\
\hline & Superior frontal gyrus & 11 & & -17 & 52 & -20 \\
\hline (C) 396-408 & (R) Postcentral gyrus (SII) & 2 & $\mathrm{E}>\mathrm{I}$ & 60 & -18 & 29 \\
\hline \multirow[t]{3}{*}{ (D) $418-430$} & (L) Rectal gyrus & 11 & $\mathrm{I}>\mathrm{E}$ & -3 & 17 & -20 \\
\hline & & 11 & & -10 & 24 & -20 \\
\hline & (R) Precentral gyrus & 6 & & 25 & -11 & 64 \\
\hline (E) $436-460$ & (R) Superior frontal gyrus & 8 & $\mathrm{~L}>\mathrm{I}$ & 25 & 24 & 50 \\
\hline
\end{tabular}

Note. First column: Time elements in milliseconds. Second column: Functional anatomical areas. Third column: Brodmann areas (BA). Forth column: Calculated contrasts (E > I: strongly increased neuronal activation during "early" compared to "intermediate" learning, I > E: strongly increased activation during "intermediate" compared to "early" learning. L > I: strongly increased activation during "late" compared to "intermediate" learning). Fifth column: Talairach coordinates of the voxel of maximal significance, in $\mathrm{mm}, p<0.05$. 


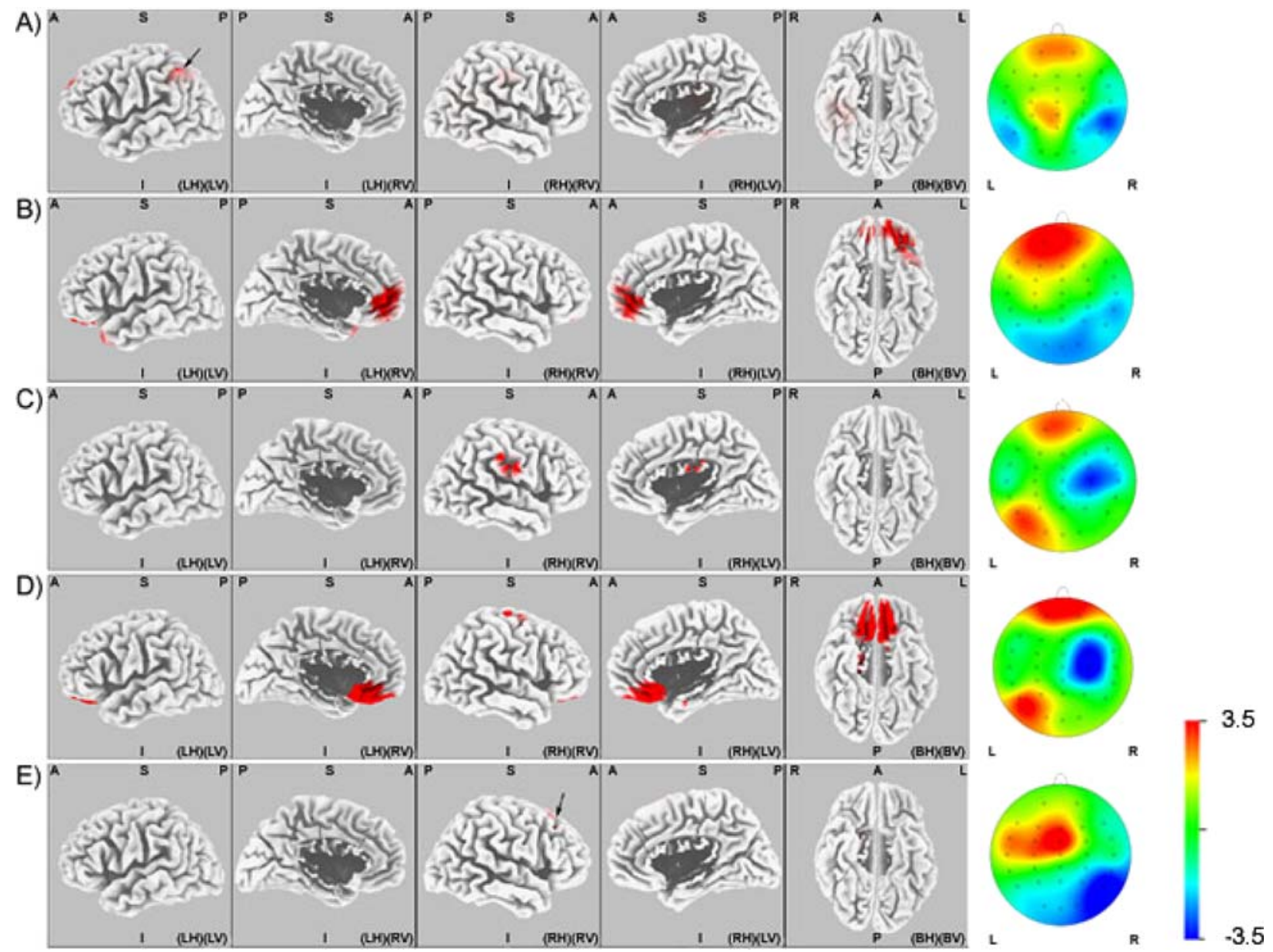

Figure 4. Localisation. Source modelling for the significantly different scalp distributions between learning stages obtained for the VEPs. LORETA-based statistical nonparametric maps (SnPM) for comparing "early" vs. "intermediate" (A-D) and "intermediate" vs. "late" learning (E). Images correspond to time elements (A) 112-154 ms, (B) 206-286 ms, (C) 396$408 \mathrm{~ms}$, (D) 418-430 ms, and (E) 436-460 ms. The right column demonstrates corresponding $t$-maps $(t=3.012, p<0.05)$. Positive $t$-values are indicated in red (increased neuronal activation during "early" compared to "intermediate" learning) and negative t-values are indicated in blue (increased neuronal activation during "intermediate" compared to "early" learning). Green indicates no statistical differences between learning stages (see color bar on the right). A = anterior. $\mathrm{P}=$ posterior. $\mathrm{S}=$ superior. $\mathrm{I}=$ inferior. $\mathrm{LH}=$ left hemisphere. $\mathrm{RH}=$ right hemisphere. $\mathrm{BH}=$ both hemispheres. $\mathrm{LV}=$ left view. $\mathrm{RV}=$ right view. $\mathrm{BV}=$ bottom view. Cortex shown in grey scale; significant activation in red $(p<0.05)$. The displays were re-computed from the KEY-LORETA software provided by Pascual-Marqui et al. (2002).

\section{LORETA Analysis of the MRPs}

The TANOVA analysis of MRPs (400 ms prior to and $500 \mathrm{~ms}$ following correct motor response) revealed increased activation during intermediate learning compared to early learning. Two time elements were detected before correct motor response and seven time elements following correct motor response. LORETA (voxel-byvoxel $t$-test) localised increased activation $360-346 \mathrm{~ms}$ before motor response bilaterally in the anterior dorsal cingulum, and 158-120 ms before motor response in the right middle frontal gyrus, partly including the dorsal premotor cortex (dPMC). Table II listed time elements and the related Talairach and Tournoux coordinates.
Figure 5 presented images corresponding to LORETA analysis and the scalp topography difference maps (as $t$-maps).

The strong neuronal activations during intermediate learning compared to early learning after the motor response were found at seven time elements ranging from an early interval at $68-80 \mathrm{~ms}$ to a late interval 410 $430 \mathrm{~ms}$. The identified brain areas constitute a network comprising right posterior cingulate gyrus $(68-80 \mathrm{~ms})$, bilaterally the anterior pole of the superior frontal gyrus extending into the OFC, left parahippocampal gyrus, left posterior cingulate gyrus (168-206 ms), left paracentral motor region including the supplementary motor area (SMA-proper, 252-298 ms), left putamen, left insula, 
Table II. LORETA analysis of the MRPs: Significant differences in brain areas between learning stages ("early" vs. "intermediate" learning; no significant differences between "intermediate" and "late" learning)

\begin{tabular}{|c|c|c|c|c|c|c|}
\hline \multirow[b]{2}{*}{ Time element (in ms) } & \multirow[b]{2}{*}{ Functional anatomic areas } & \multirow[b]{2}{*}{ BA } & \multirow[b]{2}{*}{ Contrast } & \multicolumn{3}{|c|}{ Tal coordinates } \\
\hline & & & & $x$ & $y$ & $z$ \\
\hline \multirow[t]{2}{*}{ (A) $-360--348$} & (L) Anterior cingulate & 32 & $\mathrm{I}>\mathrm{E}$ & -10 & 17 & 36 \\
\hline & (R) Anterior cingulate & 32 & & 11 & 17 & 36 \\
\hline (B) $-158--120$ & (R) Middle frontal gyrus & 6 & $\mathrm{I}>\mathrm{E}$ & 25 & 17 & 57 \\
\hline (C) $68-80$ & (R) Posterior cingulate gyrus & 23 & $\mathrm{I}>\mathrm{E}$ & 4 & -39 & 22 \\
\hline \multirow[t]{6}{*}{ (D) $168-206$} & (L) Superior frontal gyrus & 10 & $\mathrm{I}>\mathrm{E}$ & -10 & 66 & 22 \\
\hline & (L) Superior frontal gyrus & 11 & & -10 & 59 & -20 \\
\hline & (R) Superior frontal gyrus & 11 & & 11 & 59 & -20 \\
\hline & (R) Superior frontal gyrus & 11 & & 18 & 52 & -20 \\
\hline & (L) Cingulate gyrus & 31 & & -10 & -53 & 29 \\
\hline & (L) Parahippocampal gyrus & 36 & & -31 & -32 & -20 \\
\hline (E) 220-232 & (R) Middle frontal gyrus & 11 & $\mathrm{I}>\mathrm{E}$ & 25 & 45 & -13 \\
\hline \multirow{2}{*}{ (F) 252-298 } & (L) Medial frontal gyrus & 6 & $\mathrm{I}>\mathrm{E}$ & -3 & -18 & 71 \\
\hline & (R) Middle frontal gyrus & 6 & & 32 & -4 & 57 \\
\hline \multirow[t]{3}{*}{ (G) 304-320 } & (L) Putamen & & $\mathrm{I}>\mathrm{E}$ & -31 & -11 & 1 \\
\hline & (L) Insula & 13 & & -38 & -4 & 15 \\
\hline & (R) Inferior temporal gyrus & 20 & & 39 & -18 & -34 \\
\hline (H) 370-380 & (R) Inferior frontal gyrus & 9 & $\mathrm{I}>\mathrm{E}$ & 60 & 10 & 29 \\
\hline (I) $410-430$ & (R) Inferior frontal gyrus & 45 & $\mathrm{I}>\mathrm{E}$ & 53 & 10 & 22 \\
\hline
\end{tabular}

Note. Negative time elements describe significant differences detected before the motor response (A and B). For further explanations see Table I.

right inferior temporal gyrus (304-320 ms), and right inferior frontal gyrus including the ventral premotor cortex (vPMC, 370-380 ms and $410-430 \mathrm{~ms}$ ). There were no further significant differences between intermediate and late learning.

\section{Discussion}

The present study illustrates specific time intervals of neuronal modifications during visuomotor association learning. We defined three learning stages according to accuracy scores, recorded event-related potentials and performed TANOVA- and LORETA-analyses of the data to estimate the location of neuronal modifications. We used ERPs time-locked to the visual imperative stimulus (VEP) in one analysis, and ERPs time-locked to the correct motor responses (MRP) in a second analysis. Thus, we were able to estimate neural modifications associated with processing of the imperative stimuli (based on mean VEPs) independently from the activation associated with movement preparation and movement adjustment for correct associations (based on the analysis of mean MRPs). The results illustrated an increase in a parietal-prefrontal network during early learning. Advanced learning, however, is characterized by visuomotor integration- and memory-related activation that includes prefrontal, premotor, parietal, temporal, and parahippocampal activation.
We extended the conventional ERP analysis (e.g., Hammond et al. 2005) in order to calculate the intracerebral sources which are modified during visuomotor association learning by including all available electrodes. We used LORETA to estimate the intracerebral sources of EEG activity. While different analysing models have advantages and disadvantages (Michel et al. 2001), several recent studies have successfully employed LORETA as a reliable tool for localising cortical activation in various tasks ranging from visual and auditory to complex cognitive tasks (Pizzagalli et al. 2000; Vitacco et al. 2002; Esslen et al. 2004; Mulert et al. 2004a). Interestingly, the combined use of LORETA and fMRI revealed similar cortical maps to those obtained with fMRI and LORETA separately (Vitacco et al. 2002; Mulert et al. 2002). It should be noted that the significant differences of neuronal activation between the learning stages are mapped on a standardised brain model. With respect to the relatively low spatial resolution of LORETA it is supposed that the estimated sources of neuronal activation are at least in close vicinity to the mentioned anatomical regions.

\section{Stimulus-Related Activation}

Learning-related neuronal modifications were estimated on the basis of intracerebral activation associated with processing of the visual cue. These revealed 


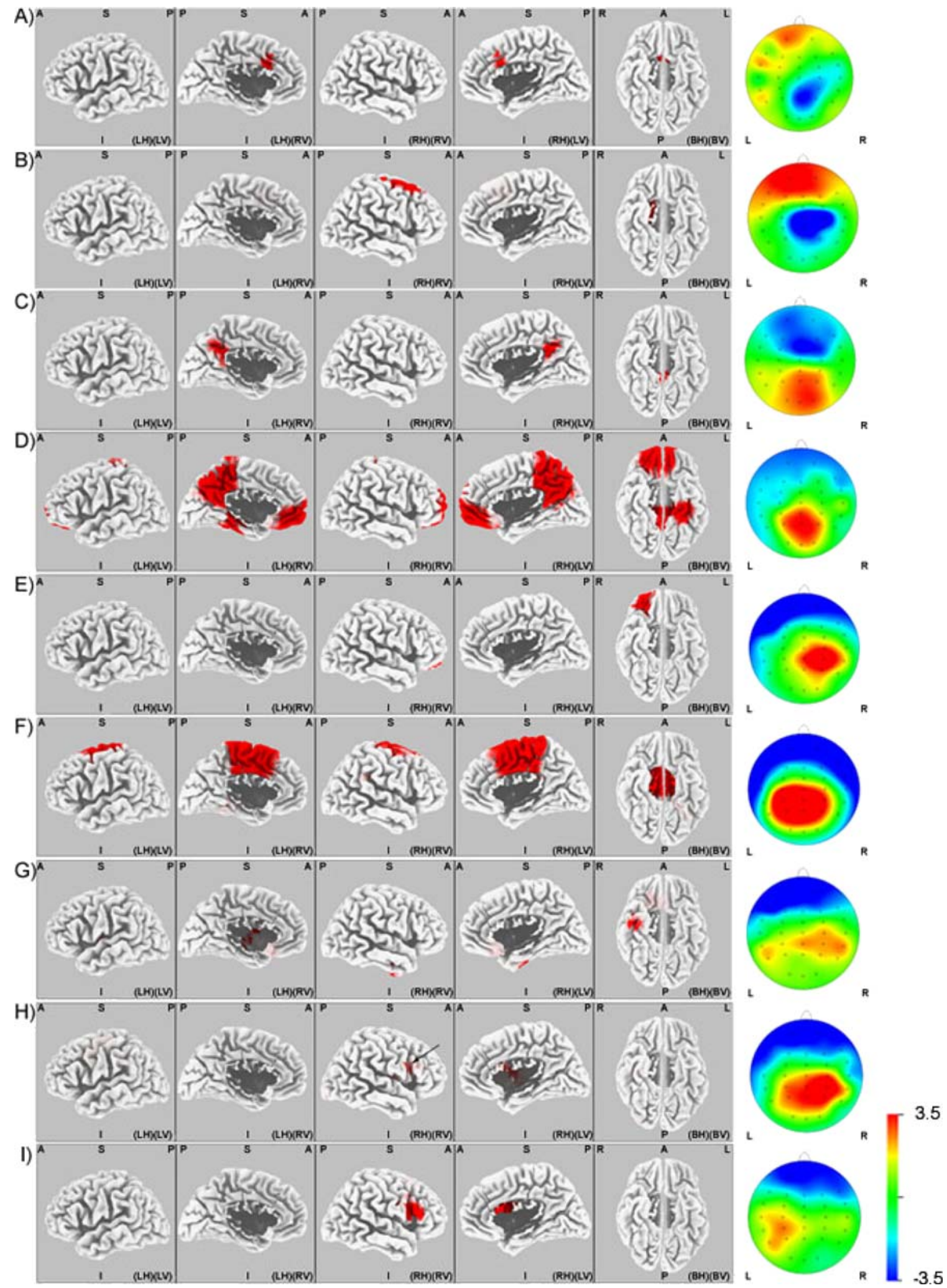

Figure 5. Localisation. Source modelling (using LORETA) for the significantly different scalp distributions between "early" and "intermediate" learning stages obtained for the MRPs. Images correspond to time elements (A) 360-348 ms and (B) $158-120 \mathrm{~ms}$ before the motor response and (C) 68-80 ms, (D) 168-206 ms, (E) 220-232 ms, (F) 252-298 ms, (G) 304-320 ms, (H) $370-380 \mathrm{~ms}$, (I) $410-430 \mathrm{~ms}$ after the motor response. Legends, colour coding, and figure orientation are the same as for Fig. 4. 
stronger intracerebral activation for the early learning stage compared to subsequent learning stages (intermediate and late) within specific time intervals and brain areas. During the early stage, stronger activation was found in the left IPL/SPL shortly after imperative stimulus presentation $(112-154 \mathrm{~ms})$ and in the right SII area $206-286 \mathrm{~ms}$ after presentation. There was also stronger intracerebral activation in the vicinity of the OFC, one within the time interval 206-286 ms (more laterally oriented with a left-sided extension into the adjacently located inferior and superior frontal gyrus) and another cluster more mesially located at the time interval 418-430 ms after the imperative stimulus.

This activation pattern most probably reflects changes in the activation of neural assemblies of the dorsal pathway (especially within IPL/SPL and SII) in association with the progression from early to intermediate learning stages. Of special interest in this context is the reduced activation in the IPL/SPL in later learning stages. The posterior IPL/SPL is involved in a variety of mental operations, including mental rotation (Jordan et al. 2001), imagining motor acts (Deiber et al. 1998), learning movement trajectories (Seitz et al. 1997), controlling self-determined finger movements (Schubert et al. 1998), and recognising objects in relation to actions (Faillenot et al. 1997). The common feature of the tasks used in these studies is the generation of a mental representation of a visual object and imagined motor acts of grasping, exploring, manipulating or manually constructing the object. This action-oriented object representation is clearly different from the object recognition processes linked with the ventral stream (Goodale et al. 1992). In visuomotor association learning, this supra-modal mental representation must be recalled, kept in mind, and then transformed into appropriate motor commands. These operations undergo a process of optimisation during learning. As a consequence, there are stronger computational demands during early learning and lesser demands during intermediate and late learning.

SII (which is more strongly activated during the early learning stage at $396-408 \mathrm{~ms}$ ) is known to be involved in several somatosensory processes ranging from pain perception to sensorimotor transformations (Inoue et al. 2002). Interestingly, SII activation is greater when sensorimotor transformation is complex or disturbed by concurrent stimulations. It is conceivable that SII is used as an additional computation resource whenever sensorimotor transformations are complex or not yet established.

We found also stronger activation during the early learning stage in a more lateral part of the left OFC 206$286 \mathrm{~ms}$ after stimulus onset, and a reversed pattern of stronger activation in the intermediate stage compared to the early learning stage in a more medial part of the OFC $418-430 \mathrm{~ms}$ after presentation of the imperative stimulus. Thus, there is a differential involvement of regions within OFC in short-term visuomotor learning as examined in our experiment. The lateral OFC is recruited for selection of correct response and inhibition of incorrect response at an early learning stage. Its stronger activation possibly reflecting greater participation of these processes at the beginning of the visuomotor association learning process. Once the association has been formed it needs to be reinforced. Greater involvement of the medial OFC has been shown in associating external (or even internal) stimuli with rewarded responses. In reviewing several neuroimaging studies, Elliott et al. (2000) have suggested that the medial OFC is critical for selecting stimuli and responses based on their reward value. The role of the medial OFC may be interpreted as activating a reward mechanism during visuomotor learning of a sensory-motor task. Alternatively, findings from Stephan et al. (2002) and Jackson et al. (2003) indicate the involvement of ventral mediofrontal cortex in subconscious movement adjustments, especially when movements are adjusted to external stimuli. Although synchronisation between movement and sensory stimuli is an essential aspect of our visuomotor association task, the medial OFC is more strongly involved in the later learning stages by "rewarding" the correct responses (second process); finer adjustments might be associated with the later learning stages.

\section{Preparation for Motor Response}

Comparison of the ERPs time-locked to the motor response (MR) and analysis of estimated intracerebral activation for the time interval before MR revealed stronger intracerebral activations in intermediate learning stages: 1. bilaterally in the anterior cingulate cortex (360-348 ms prior to MR), and 2. in the right dorsal premotor cortex (dPMC). The activation cluster in the anterior cingulate cortex (ACC) includes the mesial motor wall (SMA or CMA) and areas collectively referred to as the "cognitive" division of the cingulum. These areas are typically activated during various complex cognitive operations such as attention, error monitoring, complex reasoning, or response inhibition (Petit et al. 1996; Bush et al. 2000; Jancke et al. 2003). Which of these processes is responsible for the activations in the ACC found in our study is difficult to determine. However, it is conceivable that with increasing strength between the visual imperative stimuli and the associated motor responses the participants increase their ability to distinguish correct from incorrect associations, thus leading to stronger participation of the "cognitive" division of the cingulum. However, the cluster of activation within the mesial motor wall also extends into the CMA which is known to be involved in controlling the motivation to move or the reward associated with a particular movement (Shima 
et al. 1998). Thus, increasing proficiency in visuomotor learning of the expected reward (namely to receive a correct feedback) may well lead to stronger activations within CMA.

A further cluster indicating stronger activation during intermediate learning compared to early learning was found within the right middle frontal gyrus, including the dPMC. According to earlier hypotheses, the $\mathrm{dPMC}$ (and the ventral premotor cortex: vPMC) is specifically activated by movements that are guided by sensory information as opposed to movements that are carried out without sensory control (Halsband et al. 1985; Passingham 1988; Grafton 1994; Kawashima et al. 1995). Thus, our findings are principally in concordance with earlier data supporting the idea that the dPMC is strongly involved in visuomotor association learning (Toni et al. 1999; Toni et al. 2001). The right (ipsilateral) hemispheric activation in the dorsal PMC is consistent with results of the group of Garavan et al. (1999; Hester et al. 2004) in showing that this area might be strongly involved in the inhibitory control of actions. Inhibition of movements is a necessary consequence of visuomotor learning because incorrect visuomotor association have to be prevented. Therefore, the right dPMC could be the area where such inhibition is generated and controlled. Interestingly, several brain imaging studies found increased right-sided APMC activations during the course of motor learning, thus supporting our finding (Honda et al. 1998; Muller et al. 2002).

\section{Post-Movement Processing}

After movement execution there were stronger activations in several brain areas during the intermediate and late learning stages compared to the early learning stage. These post-movement activations correspond to results of Bender et al. (2004) and Pfurtscheller et al. (1998) by showing that motor processing is not complete and still continues after movement has stopped. This post-movement activation most likely involves processes of visuomotor integration and memory-related activation. Incoming afferent signals from the moving hand and visual signals might reflect the visual consequences of the movement. These information have to be stored and memorized for movement adjustments (such as adjusting movement parameters) that allow performance of the next movement with increased proficiency. Further, specific movements might be associated with some kind of semantic information, hence activating semantic or episodic memory information. In fact, several more or less interrelated psychological functions might become active after movement onset. In contrast to the early onset of perceptual learning, neural movement adaptations and adjustments will only start later in the course of visuomotor learning in conjunction with processes of procedural learning that most likely start relatively late. Thus, we assume the presence of movementrelated computations and resultant increases in neural activation during later learning stages in this distributed network.

In fact, several brain areas in our study showed significantly stronger activation during later learning stages, including mesial superior frontal gyrus (including SMA), OFC, parahippocampal gyrus, anterior part of the middle frontal gyrus, posterior cingulate gyrus, inferior temporal gyrus (ITG), and ventral premotor cortex (vPMC, inferior frontal gyrus). Some of the activated brain areas are part of the motor system (SMA and the $\mathrm{vPMC}$ ), while other areas are involved in various aspects of explicit learning (e.g., the parahippocampal gyrus, Epstein et al. 1998; Jordan et al. 2004), error processing or higher cognitive functions (ACC, for discussion see above), or in processes related to reward and motivation [OFC and the anterior part of the middle frontal gyrus (Heyder et al. 2004; Kringelbach et al. 2004; Rolls 2004)].

However, two classical motor areas, namely SMA and the right $\mathrm{vPMC}$, show stronger activations during advanced learning stages. These areas (and especially SMA-proper) are known to be involved in the control of complicated motor functions. SMA-proper has been shown to be involved in programming and preparing complex movements. The stronger activation within the vPMC is slightly higher but nevertheless within the range of Broca's area, as indicated by a probability map of Broca's area (Amunts et al. 2003). Evidence has been provided that a fronto-parietal network for hand-object interaction exists in humans that includes the vPMC. In addition, it has been shown that the vPMC is also involved in the preparation and imagining of movements (Jancke et al. 2001). That the right vPMC is more activated might be analogous to the stronger activation in the right-sided dPMC prior to movement onset. Motor adjustments (in the $\mathrm{APMC}$ and the vPMC) during visuomotor learning, at least in intermediate learning stages, might be specifically related to computations in motor areas ipsilateral to the moving hand.

\section{Conclusion}

Using ERPs time-locked to visual imperative stimuli and a standard method to estimate the intracerebral sources of electrical activation, we found neuronal activations in a parieto-prefrontal network, which was more strongly activated during early than advanced learning. This network was hypothesised to represent the activity of neural assemblies that are strongly involved in visual processing as well as in decision making 
for adequate motor responses. The analysis of the MRPs revealed increased activation in the anterior cingulate and right dorsal premotor cortex prior to the execution of the motor response. This activation pattern might be associated with preparation of the adequate response and inhibition of inadequate responses. Following the motor response, increased neuronal activations were detected in visuomotor integration- and memory-related regions during intermediate compared to early learning stages. Two "classical" motor areas were activated during post-movement processing including the mesial motor wall and the right vPMC. These regions are thought to use movement feedback for consolidation of the correct associations. Both, the decrease of vision-related activation and increase of motor- and memory-related activation during advanced learning clearly illustrate neuronal modifications in order to increase efficiency of visuomotor performance.

\section{Acknowledgments}

Special thanks to Dr. Roberto Pascual-Marqui for his helpful support during the EEG data analysis. This work was financed by the Swiss National Science Foundation (Project "Short-term and long-term plasticity in the sensorimotor cortex") as part of the NCCR (National Center of Competence in Research "Neural plasticity and repair") grant.

\section{References}

Amunts, K., Schleicher, A., Ditterich, A. and Zilles, K. Broca's region: Cytoarchitectonic asymmetry and developmental changes. J. Comp Neurol. 2003, 465: 72-89.

Annett, M. Five tests of hand skill. Cortex, 1992, 28: 583-600.

Bender, S., Oelkers-Ax, R., Resch, F. and Weisbrod, M. Motor processing after movement execution as revealed by evoked and induced activity. Brain Res. Cogn Brain Res. 2004, 21: 49-58.

Brasted, P.J. and Wise, S.P. Comparison of learning-related neuronal activity in the dorsal premotor cortex and striatum. Eur J Neurosci. 2004, 19(3), 721-740.

Bush, G., Luu, P. and Posner, M.I. Cognitive and emotional influences in anterior cingulate cortex. Trends Cogn Sci. 2000, 4: 215-222.

Cohen, J. Statistical power analysis for the behavioral sciences. New York: Academic Press. 1977.

Deiber, M.P., Ibanez, V., Honda, M., Sadato, N., Raman, R. and Hallett, M. Cerebral processes related to visuomotor imagery and generation of simple finger movements studied with positron emission tomography. NeuroImage. 1998, 7: 73-85.

Elliott, R., Dolan, R.J. and Frith, C.D. Dissociable functions in the medial and lateral orbitofrontal cortex: Evidence from human neuroimaging studies. Cereb. Cortex. 2000, 10: 308-317.
Epstein, R. and Kanwisher, N. A cortical representation of the local visual environment. Nature. 1998, 392: 598-601.

Esslen, M., Pascual-Marqui, R.D., Hell, D., Kochi, K. and Lehmann, D. Brain areas and time course of emotional processing. NeuroImage. 2004, 21: 1189-1203.

Faillenot, I., Toni, I., Decety, J., Gregoire, M.C. and Jeannerod, M. Visual pathways for object-oriented action and object recognition: Functional anatomy with PET. Cereb. Cortex. 1997, 7: 77-85.

Garavan, H., Ross, T.J. and Stein, E.A. Right hemispheric dominance of inhibitory control: An event-related functional MRI study. Proc. Natl. Acad. Sci. U.S.A. 1999, 96: 83018306.

Goodale, M.A. and Milner, A.D. Separate visual pathways for perception and action. Trends Neurosci. 1992, 15: 20-25.

Grafton, S.T. Cortical control of movement. Ann. Neurol. 1994, 36: 3-4.

Gray, C.M., Konig, P., Engel, A.K. and Singer, W. Oscillatory responses in cat visual cortex exhibit inter-columnar synchronization which reflects global stimulus properties. Nature. 1989, 338: 334-337.

Halsband, U. and Passingham, R.E. Premotor cortex and the conditions for movement in monkeys (Macaca fascicularis). Behav. Brain Res. 1985, 18: 269-277.

Hammond, G.R. and Fox, A.M. Electrophysiological evidence for lateralization of preparatory motor processes. Neuroreport. 2005, 16: 559-562.

Hester, R.L., Murphy, K., Foxe, J.J., Foxe, D.M., Javitt, D.C. and Garavan, H. Predicting success: Patterns of cortical activation and deactivation prior to response inhibition. J. Cogn Neurosci. 2004, 16: 776-785.

Heyder, K., Suchan, B. and Daum, I. Cortico-subcortical contributions to executive control. Acta Psychol. (Amst). 2004, 115: 271-289.

Holm, S. A simple sequentially rejective multiple test procedure. Scand J Statistics. 1979, 6, 65-70.

Honda, M., Deiber, M.P., Ibanez, V., Pascual-Leone, A., Zhuang, P. and Hallett, M. Dynamic cortical involvement in implicit and explicit motor sequence learning. A PET study. Brain. 1998, 121 (Pt 11): 2159-2173.

Inoue, K., Yamashita, T., Harada, T. and Nakamura, S. Role of human SII cortices in sensorimotor integration. Clin. Neurophysiol. 2002, 113: 1573-1578.

Jackson, P.L., Lafleur, M.F., Malouin, F., Richards, C.L. and Doyon, J. Functional cerebral reorganization following motor sequence learning through mental practice with motor imagery. NeuroImage. 2003, 20: 1171-1180.

Jackson, S.R., Jackson, G.M. and Roberts, M. The selection and suppression of action: ERP correlates of executive control in humans. Neuroreport. 1999, 10: 861-865.

Jancke, L., Kleinschmidt, A., Mirzazade, S., Shah, N.J. and Freund, H.J. The role of the inferior parietal cortex in linking the tactile perception and manual construction of object shapes. Cereb. Cortex. 2001, 11: 114-121.

Jancke, L., Specht, K., Shah, J.N. and Hugdahl, K. Focused attention in a simple dichotic listening task: An fMRI experiment. Brain Res. Cogn Brain Res. 2003, 16: 257266.

Johnson, P.B., Ferraina, S., Bianchi, L. and Caminiti, R. Cortical networks for visual reaching: Physiological and 
anatomical organization of frontal and parietal lobe arm regions. Cereb. Cortex. 1996, 6: 102-119.

Jordan, K., Heinze, H.J., Lutz, K., Kanowski, M. and Jancke, L. Cortical activations during the mental rotation of different visual objects. NeuroImage. 2001, 13: 143-152.

Jordan, K., Schadow, J., Wuestenberg, T., Heinze, H.J. and Jancke, L. Different cortical activations for subjects using allocentric or egocentric strategies in a virtual navigation task. Neuroreport. 2004, 15: 135-140.

Karni, A., Meyer, G., Rey-Hipolito, C., Jezzard, P., Adams, M.M., Turner, R. et al. The acquisition of skilled motor performance: Fast and slow experience-driven changes in primary motor cortex. Proc. Natl. Acad. Sci. U.S.A. 1998, 95: 861-868.

Kawashima, R., Roland, P.E. and O'Sullivan, B.T. Functional anatomy of reaching and visuomotor learning: A positron emission tomography study. Cereb. Cortex. 1995, 5: 111122.

Kringelbach, M.L. and Rolls, E.T. The functional neuroanatomy of the human orbitofrontal cortex: Evidence from neuroimaging and neuropsychology. Prog. Neurobiol. 2004, 72: 341-372.

Kurata, K., Tsuji, T., Naraki, S., Seino, M. and Abe, Y. Activation of the dorsal premotor cortex and pre-supplementary motor area of humans during an auditory conditional motor task. J. Neurophysiol. 2000, 84: 1667-1672.

Lehmann, D., Ozaki, H. and Pal, I. EEG alpha map series: Brain micro-states by space-oriented adaptive segmentation. Electroencephalogr. Clin. Neurophysiol. 1987, 67: 271-288.

Manly, B.F.J. (1997). Randomization, Bootstrap and Monte Carlo Methods in Biology. (second ed.) Chapman \& Hall, London.

Matelli, M. and Luppino, G. Parietofrontal circuits: Parallel channels for sensory-motor integrations. Adv. Neurol. 2000, 84: 51-61.

Matsumoto, R., Ikeda, A., Ohara, S., Matsuhashi, M., Baba, K., Yamane, F. et al. Motor-related functional subdivisions of human lateral premotor cortex: Epicortical recording in conditional visuomotor task. Clin. Neurophysiol. 2003, 114: 1102-1115.

Michel, C.M., Thut, G., Morand, S., Khateb, A., Pegna, A.J., Grave, d.P. et al. Electric source imaging of human brain functions. Brain Res. Rev. 2001, 36: 108-118.

Mulert, C., Jager, L., Pogarell, O., Bussfeld, P., Schmitt, R., Juckel, G. et al. Simultaneous ERP and event-related fMRI: Focus on the time course of brain activity in target detection. Methods Find. Exp Clin. Pharmacol. 2002, 24 Suppl D: $17-20$.

Mulert, C., Jager, L., Schmitt, R., Bussfeld, P., Pogarell, O., Moller, H.J. et al. Integration of fMRI and simultaneous EEG: Towards a comprehensive understanding of localization and time-course of brain activity in target detection. NeuroImage. 2004a, 22: 83-94.

Mulert, C., Pogarell, O., Juckel, G., Rujescu, D., Giegling, I., Rupp, D. et al. The neural basis of the P300 potential. Focus on the time-course of the underlying cortical generators. Eur Arch. Psychiatry Clin. Neurosci. 2004b, 254: 190-198.

Muller, R.A., Kleinhans, N., Pierce, K., Kemmotsu, N. and Courchesne, E. Functional MRI of motor sequence acquisi- tion: Effects of learning stage and performance. Brain Res. Cogn Brain Res. 2002, 14: 277-293.

Nakamura, K., Sakai, K. and Hikosaka, O. Neuronal activity in medial frontal cortex during learning of sequential procedures. J Neurophysiol. 1998, 80: 2671-2687.

Nichols, T.E. and Holmes, A.P. Nonparametric permutation tests for functional neuroimaging: A primer with examples. Hum. Brain Mapp. 2002, 15: 1-25.

Pascual-Marqui, R.D., Esslen, M., Kochi, K. and Lehmann, D. Functional imaging with low-resolution brain electromagnetic tomography (LORETA): A review. Methods Find. Exp. Clin. Pharmacol. 2002, 24 Suppl C: 91-95.

Passingham, R.E. Premotor cortex and preparation for movement. Exp. Brain Res. 1988, 70: 590-596.

Petit, L., Orssaud, C., Tzourio, N., Crivello, F., Berthoz, A., and Mazoyer, B. Functional anatomy of a prelearned sequence of horizontal saccades in humans. J. Neurosci. 1996, 16: 3714-3726.

Pfurtscheller, G., Zalaudek, K. and Neuper, C. Event-related beta synchronization after wrist, finger and thumb movement. Electroencephalogr. Clin. Neurophysiol. 1998, 109: 154-160.

Pizzagalli, D., Lehmann, D., Koenig, T., Regard, M. and Pascual-Marqui, R.D. Face-elicited ERPs and affective attitude: Brain electric microstate and tomography analyses. Clin. Neurophysiol. 2000, 111: 521-531.

Rolls, E.T. The functions of the orbitofrontal cortex. Brain Cogn. 2004, 55: 11-29.

Rosenbaum, D.A. Human movement initiation: Specification of arm, direction, and extent. J. Exp. Psychol. Gen. 1980, 109: 444-474.

Sakai, K., Hikosaka, O., Miyauchi, S., Sasaki, Y., Fujimaki, N. and Putz, B. Presupplementary motor area activation during sequence learning reflects visuo-motor association. J. Neurosci. 1999, 19: RC1.

Schubert, T., von Cramon, D.Y., Niendorf, T., Pollmann, S. and Bublak, P. Cortical areas and the control of self-determined finger movements: An fMRI study. Neuroreport. 1998, 9: 3171-3176.

Seitz, R.J., Canavan, A.G., Yaguez, L., Herzog, H., Tellmann, L., Knorr, U. et al. Representations of graphomotor trajectories in the human parietal cortex: Evidence for controlled processing and automatic performance. Eur J Neurosci. 1997, 9: 378-389.

Shima, K. and Tanji, J. Role for cingulate motor area cells in voluntary movement selection based on reward. Science. 1998, 282: 1335-1338.

Silva, L.R., Amitai, Y. and Connors, B.W. Intrinsic oscillations of neocortex generated by layer 5 pyramidal neurons. Science. 1991, 251: 432-435.

Sinai, A. and Pratt, H. High-resolution time course of hemispheric dominance revealed by low-resolution electromagnetic tomography. Clin. Neurophysiol. 2003, 114: 1181-1188.

Staines, W.R., Padilla, M. and Knight, R.T. Frontal-parietal event-related potential changes associated with practising a novel visuomotor task. Brain Res Cogn Brain Res. 2002, 13: 195-202.

Stephan, K.M., Thaut, M.H., Wunderlich, G., Schicks, W., Tian, B., Tellmann, L. et al. Conscious and subconscious 
sensorimotor synchronization-prefrontal cortex and the influence of awareness. NeuroImage. 2002, 15: 345-352.

Strik, W.K., Fallgatter, A.J., Brandeis, D. and Pascual-Marqui, R.D. Three-dimensional tomography of event-related potentials during response inhibition: Evidence for phasic frontal lobe activation. Electroencephalogr. Clin. Neurophysiol. 1998, 108: 406-413.

Thoenissen, D., Zilles, K. and Toni, I. Differential involvement of parietal and precentral regions in movement preparation and motor intention. J. Neurosci. 2002, 22: 9024-9034.

Toni, I., Ramnani, N., Josephs, O., Ashburner, J. and Passingham, R.E. Learning arbitrary visuomotor associations: Temporal dynamic of brain activity. NeuroImage 2001, 14 : 1048-1057.

Toni, I., Rowe, J., Stephan, K.E. and Passingham, R.E. Changes of cortico-striatal effective connectivity during visuomotor learning. Cereb. Cortex. 2002a, 12: 1040-1047.
Toni, I., Schluter, N.D., Josephs, O., Friston, K. and Passingham, R.E. Signal-, set- and movement-related activity in the human brain: An event-related fMRI study. Cereb. Cortex. 1999, 9: 35-49.

Toni, I., Shah, N.J., Fink, G.R., Thoenissen, D., Passingham, R.E. and Zilles, K. Multiple movement representations in the human brain: An event-related fMRI study. J. Cogn Neurosci. 2002b, 14: 769-784.

Vitacco, D., Brandeis, D., Pascual-Marqui, R. and Martin, E. Correspondence of event-related potential tomography and functional magnetic resonance imaging during language processing. Hum. Brain Mapp. 2002, 17: 412.

Wise, S.P., Boussaoud, D., Johnson, P.B. and Caminiti, R. Premotor and parietal cortex: Corticocortical connectivity and combinatorial computations. Annu. Rev. Neurosci. 1997, 20: $25-42$. 